\title{
BMJ Open Prevalence of Alcohol Consumption in Emergency department presentations (PACE) in Queensland, Australia, using alcohol biomarkers ethanol and phosphatidylethanol: an observational study protocol
}

Kim A Vuong (D) ,1,2 Silvia Manzanero (D) ,3,4 Jacobus P J Ungerer (D) ,,6 Gary Mitchell, ${ }^{7,8}$ Brett McWhinney, ${ }^{5}$ Kirsten Vallmuur (1) , ${ }^{1,3}$ Jacelle Warren (1) ,1,3 Victoria McCreanor (D) , ${ }^{1,3}$ Tegwen Howell, ${ }^{9}$ Clifford Pollard, ${ }^{3}$ Michael Schuetz, ${ }^{3,4}$ Anna Zournazi, ${ }^{5}$ Cate M Cameron (D) ${ }^{1,3}$

To cite: Vuong KA, Manzanero S, Ungerer JPJ, et al. Prevalence of Alcohol Consumption in Emergency department presentations (PACE) in Queensland, Australia, using alcohol biomarkers ethanol and phosphatidylethanol: an observational study protocol. BMJ Open 2021;11:e047887. doi:10.1136/ bmjopen-2020-047887

- Prepublication history for this paper is available online. To view these files, please visit the journal online (http://dx.doi org/10.1136/bmjopen-2020047887).

Received 14 December 2020 Accepted 12 October 2021

Check for updates

(C) Author(s) (or their employer(s)) 2021. Re-use permitted under CC BY-NC. No commercial re-use. See rights and permissions. Published by BMJ.

For numbered affiliations see end of article.

Correspondence to

Dr Kim A Vuong;

kim.anhvuong@qut.edu.au

\section{ABSTRACT}

Introduction Alcohol use in patients presenting to the emergency department (ED) is a significant problem in many countries. There is a need for valid and reliable surveillance of the prevalence of alcohol use in patients presenting to the ED, to provide a more complete picture of the risk factors and inform targeted public health interventions. This PACE study will use two biomarkers, blood ethanol and phosphatidylethanol (PEth), to determine the patterns, presence and level of alcohol use in patients presenting to an Australian ED.

Methods and analysis This is an observational prevalence study involving the secondary use of routinely collected blood samples from patients presenting to the Royal Brisbane and Women's Hospital (RBWH) Emergency and Trauma Centre (ETC). Samples will be tested for acute and medium-term alcohol intake using the two biomarkers blood ethanol and PEth respectively, over one collection period of 10-12 days. Descriptive statistics such as frequencies, percentages, means, SD, medians and IQRs, will be used to describe the prevalence, pattern and distribution of acute and medium-term alcohol intake in the study sample. The correlation between acute and medium-term alcohol intake levels will also be examined. Ethics and dissemination This study has been approved by the RBWH Human Research Ethics Committee (reference, LNR/2019/QRBW/56859). Findings will be disseminated to key stakeholders such as RBWH ETC, Australasian College for Emergency Medicine, Royal Australasian College of Surgeons, Statewide Clinical Networks, and used to inform clinicians and hospital services. Findings will be submitted for publication in peer-reviewed journals and presentation at appropriate conferences.

\section{INTRODUCTION}

Harmful alcohol use is one of the leading risk factors for population health worldwide. ${ }^{1}$
Strengths and limitations of this study

A novel surveillance approach comparing acute and medium-term alcohol use in patients presenting to the emergency department.

- Institutional approval for a waiver of consent was obtained which addresses the limitations of other studies such as low consent rates, self-report bias and resource intensive data collection methods.

- Objective data collection method.

- Clinical care received by patients will not be affected as staff will not be informed of the study details and will not know the sampling dates.

- Data will only be collected during a single sampling period of 10-12 days due to budget constraints.

In Australia, harmful alcohol use results in more than 144000 hospitalisations and 70 000 victims of alcohol-related assaults, with 24000 attributed to alcohol-related domestic violence. These harms are estimated to cost the nation $\$ 36$ billion annually. ${ }^{2}$ Alcohol-involved presentations to the emergency department (ED) constitute a significant public health problem in many countries such as the USA, ${ }^{3}$ Ireland $^{4}$ and Australia, ${ }^{5} 6$ impacting ED workloads, ${ }^{5}$ patient management and treatment, ${ }^{5}$ as well as having considerable safety implications for first responders and ED staff. ${ }^{56}$ Routine collection of alcohol data in ED presentations would enable trend monitoring, identify contributing risk factors at hospital presentation, and inform the development of appropriate public health interventions. For instance, the ability to monitor trends in alcohol-related ED presentations 
was demonstrated in the USA using a nationwide dataset, the Nationwide Emergency Department Sample, which records acute and chronic alcohol-related data. ${ }^{7}$ Despite these potential advantages, in Queensland, Australia there is no objective measurement of alcohol and other drug involvement in ED presentations. ${ }^{8}$ The rationale against routinely testing for, or recording, alcohol presence in patients in the ED, especially patients with altered levels of consciousness, primarily stems from the potential to bias clinical decision making or prematurely ruling out differential diagnoses due to a presumption that the patient's condition is due to acute alcohol consumption. ${ }^{9}$

Blood ethanol, the most frequently used biomarker of alcohol use, may not always accurately reflect the patient's ethanol exposure. Due to its relatively short half-life in blood, it is only a snapshot of the blood alcohol level at that moment in time. ${ }^{10}{ }^{11}$ For a complete picture of the impact of alcohol use in ED presentations, patients with medium or long-term high alcohol consumption must also be identified. This is currently not possible without the use of screening tools and patient self-report surveys, both with limitations. ${ }^{12-15}$ A relatively new test for the biomarker phosphatidylethanol (PEth), with excellent sensitivity and specificity, ${ }^{10}{ }^{16}$ may provide more reliable detection of alcohol intake than blood ethanol. As a marker of medium-term and long-term alcohol use, PEth levels can indicate a person's 'average' alcohol consumption during the previous 2-4 weeks. ${ }^{10} 1117$ As such, the PEth test could be the tool required to quantify medium to long-term alcohol exposure and its impact on hospital presentations.

Routine blood alcohol testing has potential value for service provision and risk management in the $\mathrm{ED}$, in addition to providing evidence for prevention in public health. ${ }^{18}$ Identifying medium and long-term alcohol use in patients would also improve our current understanding of intoxication (immediate vs longer-term consumption) among ED presentations. Using both blood ethanol and PEth as alcohol markers, this PACE study addresses the gap in knowledge about the prevalence of both immediate alcohol consumption and longer-term alcohol use in patients who present to the ED.

\section{Methods and analysis}

Study design

Observational prevalence study.

This study involves the secondary use of blood and serum samples, collected from patients presenting to the Royal Brisbane and Women's Hospital (RBWH) Emergency and Trauma Centre (ETC) in Brisbane, Queensland, Australia. From herein, ETC will be referred to as ED. The RBWH is a tertiary referral hospital and is one of the busiest hospitals in the State, treating local patients as well as patients from other States within a 2200 $\mathrm{km}$ radius. The RBWH ED sees the largest volume of patients annually in Australia with over 82000 presentations. ${ }^{19}$ The design of this study, summarised in figure 1 , will include approximately 975 blood samples collected

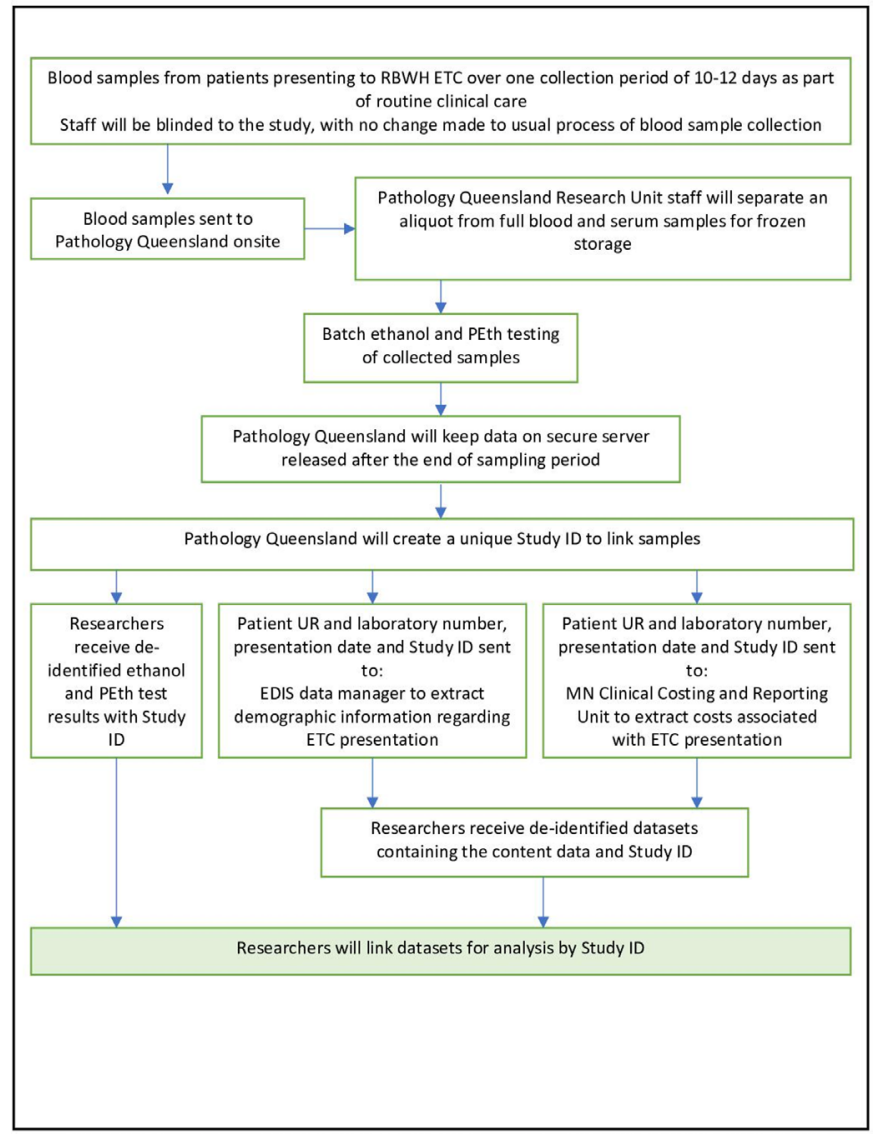

Figure 1 The collection of blood samples from patients presenting to the Royal Brisbane and Women's Hospital (RBWH) Emergency and Trauma Centre (ETC) study design. EDIS, Emergency Department Information System; ID, identification.

over a 10 to 12-day period, to be tested for both alcohol exposure markers: blood ethanol and PEth.

The RBWH ED staff will be aware of the study, but will not be informed of the details and will not know the dates during which sampling will occur. This is to prevent the occurrence of a Hawthorne effect, ${ }^{20}$ where staff might change the frequency of blood testing during the study period if aware of the specifics of the study. No change will be made to the usual care of patients presenting to the ED. The study blood ethanol and PEth test results will be protected from release until the sampling period has ended to ensure results do not affect the clinical management of patients.

\section{Sample size}

Approximately $40 \%$ of patients presenting to the RBWH ED have blood samples taken during their presentation, usually including a full blood count and other biochemistry testing as part of routine clinical care, resulting in 950-1000 samples potentially available for testing during a 1-week period (MN Clinical Costing and Reporting Unit, RBWH ETC Presentations 2019). To enable coverage of all days of the week, and allow for the likelihood of missed samples, a minimum total of 975 samples will be obtained over the study period. 
Blood sample collection and storage

RBWH ED blood samples are routinely sent to Pathology Queensland for processing. During the study period, Pathology Queensland Research Unit staff will intercept and separate an aliquot from the full blood and serum samples for frozen storage to subsequently undertake batch ethanol and PEth testing. Pathology Queensland will create a unique Study ID and linkage key which will be kept on a secure server. For each sample, Pathology Queensland will record details such as patient unit record number, laboratory number, presentation date and Study ID which will be sent to the ED data manager to extract demographic and clinical information. Blood samples will be stored at $-80^{\circ} \mathrm{C}$, the recommended temperature to ensure stability. ${ }^{21}$ Testing will be conducted eight to twelve weeks after the sampling period.

\section{Blood sample testing methods}

A validated ultra-performance liquid chromatography tandem mass spectrometry method for quantification of PEth 16:0/18:1, 16:0/18:2, 18:1/18:1 in the range 0-2000 $\mathrm{\mu g} / \mathrm{L}$ will be used. The PEth 16:0/18:1, 16:0/18:2, $18: 1 / 18: 1$ and the internal standard (IS) phosphatidylmethanol 18:1/18:1 $(250 \mu \mathrm{g} / \mathrm{L})$, are extracted from whole blood $(150 \mu \mathrm{L})$ by simple protein precipitation with 2-propanol $(450 \mu \mathrm{L})$. Chromatography is achieved using an Acquity BEH-C8 $(2.1 \times 50 \mathrm{~mm}, 1.7 \mu \mathrm{m})$ column. PEth concentration will be measured by a Waters TQS mass spectrometer (Water Corporation, Milford, Massachusetts, USA) operating in negative mode with multiple reaction monitoring for specific transitions for each analyte. The coefficient of variation (CV), as an indication of within-assay and between-assay imprecision, is $\leq 8.0 \%$ and the limit of blank is $1.0 \mu \mathrm{g} / \mathrm{L}$. Recoveries are $98 \%-102 \%(\mathrm{CV} \leq 4.0 \%)$. Repeated freezing $\left(-80^{\circ} \mathrm{C}\right)$ and thawing does not affect the concentration. ${ }^{22}$ Serum ethanol will be measured by an enzymatic rate assay on Beckman Coulter Synchron Clinical Systems. ${ }^{23}$ Withinassay and between-assay CV is $\leq 10.0 \%$ across two levels of ethanol 11.9 and $32.0 \mathrm{mmol} / \mathrm{L}$.

\section{Data collection}

Data will be collected over one collection period of 10-12 days and will include a public holiday weekend, working week and a non-public holiday weekend. To minimise the impact of the current COVID-19 pandemic on results, data will not be collected during any period when there is a locally impacting lockdown or coronavirus outbreak. For all patients presenting to the ED during the study period (including those who have blood samples collected and those who do not), demographic, clinical presentation and costing data will be internally extracted from the Emergency Department Information System and the health services administrative database.

Demographic factors such as age, gender, identifying as Aboriginal and/or Torres Strait Islander, and postcode of usual residence will be extracted. Clinical information will include date and time of presentation and discharge, the presenting problem and nurse assessment fields (free text), principal diagnosis code (International Classification of Diseases, Tenth Edition, Australian Modification), presenting complaint code, funding source and whether the patient was admitted to hospital. Only de-identified test results and linked extracted datasets will be returned to the study researchers for analysis (figure 1).

\section{Data analysis}

The primary analysis will be to:

1. Describe the prevalence, pattern and distribution of alcohol and PEth levels in the study sample over the inclusion period. Prevalence estimates will include 95\% confidence limits based on the Wilson method. ${ }^{24}$

Cases will be described by clinical presentation using the principal diagnosis groups as well as demographic distributions using appropriate descriptive statistics (frequencies, percentages, means, SD, medians and IQRs). Since the COVID-19 context is likely to impact presentations to the ED, COVID-19-related presentations will be reported and excluded from the results.

The secondary analysis will:

2. Assess the correlation between blood ethanol and PEth results using the Spearman method.

3. Describe the association between test results and alcohol-related ICD coded primary diagnoses in the clinical data.

4. Compare the demographic and clinical characteristics of the study sample with the patients who present to the ED during the same period but do not undergo blood sampling or whose samples are not redirected for study testing. These comparisons will determine how representative the sample is to the population, thus informing the generalisability of the findings.

The blood alcohol concentration (BAC) levels will be guided by the BAC cut-offs and associated short-term effects on an Australian Government website by the Department of Health. ${ }^{25}$ BAC levels are categorised as: light: BAC 0.01 to $<0.05$; significant: BAC 0.05 to 0.30 and potential coma/death: BAC $>0.30$.

PEth levels are categorised according to Ulwelling and Smith ${ }^{11}$ : light or no consumption $(<20 \mathrm{ng} / \mathrm{mL})$; significant consumption (20-200 ng/mL) and heavy consumption $(>200 \mathrm{ng} / \mathrm{mL})$.

\section{Patient and public involvement}

This study relies solely on the secondary use of routinely collected blood and serum samples from patients presenting to the ED, as such, there will be no direct patient involvement in this study.

\section{DISCUSSION}

Alcohol use in patients presenting to the ED is a significant public health problem and yet little is known about the prevalence of both acute and longer-term alcohol exposure in the context of hospital presentations and health service use. To monitor trends and respond with 
appropriate public health interventions, there is a need for valid and reliable indicators of alcohol use in patients presenting to the ED. Despite there being a growing body of research on alcohol-involved presentations in EDs in Australia, ${ }^{5-7} 12-152627$ many of these studies have been point-prevalence surveys based on screening tools and patient self-report. ${ }^{12-15}$ These studies are resourceintensive, ${ }^{12-15}$ sometimes requiring dedicated staff on-site in the ED for 24-hour time periods to enable comprehensive patient screening, consent and interviews. Furthermore, low consent rates bias study validity, and data collection based on self-reporting of risky behaviour has been shown to result in flawed measurements and an underestimation of the true prevalence. ${ }^{13142829}$

To address these methodological challenges, a waiver of consent was obtained from our institution's research ethics committee for the secondary testing of blood samples obtained from routine clinical care in patients presenting to the ED. In addition to the waiver of consent, approval was obtained for the release of confidential information for the purposes of research under the provision of section 280 of Public Health Act 2005, Queensland. Obtaining a waiver of consent is a key approach and strength of this study as it allows for the collection of objective data on the presence and levels of alcohol use in patients presenting to the ED. Additionally, removing the need for individual consent will reduce bias and minimise the resource intensity and logistical challenges of previous studies. ${ }^{13} 1428$ Finally, this study will not affect the clinical care participants receive, because care providers will not be aware of the purpose of the study nor be able to access the test results.

Another novel approach of this study is the comparison of immediate and ongoing alcohol use indicated in ED presentations. Ethanol has a relatively short half-life in blood, ${ }^{1011}$ and levels may not always accurately reflect the alcohol exposure relevant to a patient's presentation to the ED. Although the use of PEth tests in determining alcohol consumption in EDs is limited (one other study known), ${ }^{17}$ studies have demonstrated more specificity and sensitivity compared with other biomarkers for alcohol use. $^{1016}$

The availability of reliable data on alcohol use and levels could provide a more complete picture of the risk factors present when patients attend the ED, enable early recognition and intervention and inform both clinical practice guidelines and targeted public health interventions and monitoring. Further, findings from this study may be used to compare the prevalence of alcohol use to other studies that are similar in context (ie, prevalence of alcohol using breath tests). ${ }^{12}$ The use of the novel approach taken in this study will address the limitations of other study methods, such as low consent rates, self-report bias and resource intensive data collection methods. While this novel method of using both blood ethanol and PEth biomarkers is promising, there are some limitations that should be addressed. Results that use the proposed alcohol consumption categories (light, significant, heavy) in this study should be interpreted with care, as a recent short period of heavy drinking may display the same reading as ongoing heavy drinking. Therefore, the information from blood ethanol tests in conjunction with available clinical data (eg, primary diagnosis of alcohol gastritis) may provide a more complete picture of the findings than PEth levels alone. Further, a limitation of all long-term markers is that they do not accurately reflect the pattern of blood concentrations over time. Although PEth levels may not accurately reflect the pattern of ethanol intake, PEth levels are directly proportional to the concentration of ethanol and the time of exposure, ${ }^{1730}$ and PEth levels can distinguish between abstinence, moderate and high ethanol intake. As no perfect marker exists to monitor and/or quantify the use of alcohol, ${ }^{17} 3132$ each marker has limitations, and results should be interpreted within the limitations of each marker used. A second limitation is that it may not be cost-effective to conduct all year round, but rather, could be used for periodic sampling to monitor trends. For example, testing could be conducted during certain holiday periods or weekends when alcohol consumption is more prevalent. Such information could be used to inform targeted public health interventions to reduce harmful alcohol use. If this methodological approach were to be used in the future for periodic sampling to monitor trends, future research could undertake consultation with patients and/or clinicians to examine any potential concerns about the secondary use of blood samples.

\section{Author affiliations}

${ }^{1}$ Australian Centre for Health Services Innovation (AusHSI), Centre for Healthcare Transformation, Queensland University of Technology, Brisbane, Queensland, Australia

${ }^{2}$ Centre for Accident Research and Road Safety - Queensland (CARRS-Q), Queensland University of Technology, Brisbane, Queensland, Australia

${ }^{3}$ Jamieson Trauma Institute, Royal Brisbane \& Women's Hospital (RBWH), Metro North Health, Brisbane, Queensland, Australia

${ }^{4}$ School of Clinical Sciences, Queensland University of Technology, Brisbane, Queensland, Australia

${ }^{5}$ Chemical Pathology, Pathology Queensland, Queensland Health, Royal Brisbane \& Women's Hospital (RBWH), Metro North Health, Brisbane, Queensland, Australia ${ }^{6}$ Faculty of Biomedical Science, The University of Queensland, Brisbane,

Queensland, Australia

${ }^{7}$ Royal Brisbane \& Women's Hospital (RBWH), Metro North Health, Brisbane, Queensland, Australia

${ }^{8}$ School of Medicine, The University of Queensland, Brisbane, Queensland, Australia ${ }^{9}$ Remote Resolve, Toowong, Queensland, Australia

Contributors CMC planned the project, sought ethics, data custodian approvals and grant funding, and reviewed and approved the final manuscript. SM contributed to the planning of the study design and provided feedback on the manuscript. KAV drafted the initial manuscript. VM contributed to the study planning and provided feedback on the manuscript. KV contributed to the study planning and provided feedback on the manuscript. JW contributed to the study plan and provided feedback on the manuscript. GM contributed to study design, clinical impact and feedback on the manuscript. JPJU and BM contributed to the overall study plan and designed the sample processing methodology. AZ contributed to internal laboratory processes and logistics for blood sample collection and has provided feedback on the manuscript. TH contributed to the planning of the study design and provided feedback on the manuscript. CP and MS contributed to the study plan and grant funding application. All coauthors reviewed and approved the final manuscript. 
Funding This study has received project funding from the Jamieson Trauma Institute, Royal Brisbane and Women's Hospital. The Australian Centre for Health Services Innovation provided funding for the publication of this paper.

\section{Competing interests None declared.}

Patient and public involvement Patients and/or the public were not involved in the design, or conduct, or reporting, or dissemination plans of this research.

\section{Patient consent for publication Not applicable.}

Ethics approval This study has been approved by the Royal Brisbane and Women's Hospital Human Research Ethics Committee (reference, LNR/2019/ QRBW/56859). The study will be conducted in compliance with the National Statement on Ethical Conduct in Humans: General requirements for consent (2.2). A waiver of consent was approved in accordance with section 2.3.10 of the National Statement, to ensure that the validity of the study results was not compromised by a low or significantly biased consent rate. Approval was obtained for the release of confidential information for the purposes of research under the provision of section 280 of Public Health Act 2005, Queensland. Findings will be disseminated to key stakeholders such as RBWH ETC, Australasian College for Emergency Medicine (ACEM), Royal Australasian College of Surgeons (RACS), Statewide Clinical Networks as well as informing clinicians and hospital services. Findings will be submitted for publication in peer-reviewed journals and presentation at conferences.

Provenance and peer review Not commissioned; externally peer reviewed.

Open access This is an open access article distributed in accordance with the Creative Commons Attribution Non Commercial (CC BY-NC 4.0) license, which permits others to distribute, remix, adapt, build upon this work non-commercially, and license their derivative works on different terms, provided the original work is properly cited, appropriate credit is given, any changes made indicated, and the use is non-commercial. See: http://creativecommons.org/licenses/by-nc/4.0/.

\section{ORCID iDs}

Kim A Vuong http://orcid.org/0000-0002-2611-2698

Silvia Manzanero http://orcid.org/0000-0002-5294-7082

Jacobus P J Ungerer http://orcid.org/0000-0003-4929-0929

Kirsten Vallmuur http://orcid.org/0000-0002-3760-0822

Jacelle Warren http://orcid.org/0000-0002-2273-1846

Victoria McCreanor http://orcid.org/0000-0002-0589-8521

Cate M Cameron http://orcid.org/0000-0003-1476-5744

\section{REFERENCES}

1 World Health Organization. Global status report on alcohol and health 2018. Geneva: World Health Organization, 2018. https://www.who. int/substance_abuse/publications/global_alcohol_report/en/

2 Foundation for Alcohol Research and Education. Stopping harm caused by alcohol. Available: https://fare.org.au/about/ [Accessed Apr 2020].

3 Mullins PM, Mazer-Amirshahi M, Pines JM. Alcohol-Related visits to US emergency departments, 2001-2011. Alcohol Alcohol 2017;52:119-25.

4 McNicholl B, Goggin D, O'Donovan D. Alcohol-related presentations to emergency departments in Ireland: a descriptive prevalence study. BMJ Open 2018;8:e021932-6.

5 Egerton-Warburton D, Gosbell A, Wadsworth A, et al. Perceptions of Australasian emergency department staff of the impact of alcoholrelated presentations. Med J Aust 2016;204:155

6 Gilchrist H, Jones SC, Barrie L. Experiences of emergency department staff: alcohol-related and other violence and aggression. Australasian Emergency Nursing Journal 2011;14:9-16.

7 White AM, Slater ME, Ng G, et al. Trends in alcohol-related emergency department visits in the United States: results from the nationwide emergency department sample, 2006 to 2014. Alcohol Clin Exp Res 2018;42:352-9.

8 Vallmuur K, Limbong J, Barker R, et al. A comparison of methods to identify alcohol involvement in youth injury-related emergency department presentation data. Drug Alcohol Rev 2013;32:519-26.

9 Lucchiari C, Pravettoni G. The role of patient involvement in the diagnostic process in internal medicine: a cognitive approach. Eur $J$ Intern Med 2013;24:411-5.
10 Aradottir S, Asanovska G, Gjerss S, et al. Phosphatidylethanol (PetH) concentrations in blood are correlated to reported alcohol intake in alcohol-dependent patients. Alcohol Alcohol 2006;41:431-7.

11 Ulwelling W, Smith $\mathrm{K}$. The petH blood test in the security environment: what it is; why it is important; and interpretative guidelines. J Forensic Sci 2018;63:1634-40.

12 Egerton-Warburton D, Gosbell A, Wadsworth A, et al. Survey of alcohol-related presentations to Australasian emergency departments. Med J Aust 2014;201:584-7.

13 Browne AL, Newton M, Gope M, et al. Screening for harmful alcohol use in Australian trauma settings. Injury 2013;44:110-7.

14 Howard R, Fry S, Chan A, et al. A feasible model for early intervention for high-risk substance use in the emergency department setting. Aust Health Rev 2019;43:188-93.

15 McLay SV, MacDonald E, Fatovich DM, et al. Alcohol-Related presentations to the Royal Perth hospital emergency department: a prospective study. Emerg Med Australas 2017;29:531-8.

16 Hartmann S, Aradottir S, Graf M, et al. Phosphatidylethanol as a sensitive and specific biomarker: comparison with gamma-glutamyl transpeptidase, mean corpuscular volume and carbohydratedeficient transferrin. Addict Biol 2007;12:81-4.

17 Viel G, Boscolo-Berto R, Cecchetto G. Phosphatidylethanol in blood as a marker of chronic alcohol use: a systematic review and metaanalysis. Int J Mol Sci 2012;13:14788-812.

18 Flewellen RW. Behavioural intention to provide screening and brief intervention for alcohol-related injury in the emergency department: a guiding framework using the theory of planned behaviour [dissertation on the internet. Perth, WA: Curtin University, 2017. https://espace.curtin.edu.au/handle/20.500.11937/59108

19 et alBrisbane R, Hospital Women's, Hospital MN. Available: https:// metronorth.health.qld.gov.au/rbwh/about-us [Accessed July 2020].

20 McCambridge J, Witton J, Elbourne DR. Systematic review of the Hawthorne effect: new concepts are needed to study research participation effects. J Clin Epidemiol 2014;67:267-77.

21 Faller A, Richter B, Kluge M, et al. Stability of phosphatidylethanol species in spiked and authentic whole blood and matching dried blood spots. Int J Legal Med 2013;127:603-10.

22 Andreassen TN, Havnen H, Spigset O, et al. High throughput UPLC $($-MSMS method for the analysis of phosphatidylethanol (PetH) 16:0/18:1, a specific biomarker for alcohol consumption, in whole blood. J Anal Toxicol 2018;42:33-41.

23 Coulter B. Synchron system(s) chemistry information sheet. Beckman Coulter, Inc, 2010: 11 p. https://www.beckmancoulter.com/wsrportal/ techdocs?docname=cis/A18490/AF/EN_ETOH.pdf

24 Brown LD, Cai TT, DasGupta A. Interval estimation for a binomial proportion. Statist. Sci. 2001;16:101-33.

25 Department of Health. What are the effects of alcohol? Canberra, ACT: Department of Health, 2020. https://www.health.gov.au/ health-topics/alcohol/about-alcohol/what-are-the-effects-ofalcohol

26 Kisely S, Lawrence D. A time series analysis of alcohol-related presentations to emergency departments in Queensland following the increase in alcopops tax. $J$ Epidemiol Community Health 2016;70:181-6.

27 Gale M, Muscatello DJ, Dinh M, et al. Alcopops, taxation and harm: a segmented time series analysis of emergency department presentations. BMC Public Health 2015;15:468.

28 Sun W, Jian L, Xiao J, et al. The impact of alcohol restriction on hospital and emergency department service utilizations in two remote towns in the Kimberley region of Western Australia. Front Public Health 2019;7:17.

29 Davis CG, Thake J, Vilhena N. Social desirability biases in self-reported alcohol consumption and harms. Addict Behav 2010;35:302-11.

30 Nalesso A, Viel G, Cecchetto G, et al. Quantitative profiling of phosphatidylethanol molecular species in human blood by liquid chromatography high resolution mass spectrometry. J Chromatogr $A$ 2011;1218:8423-31.

31 Biondi A, Freni F, Carelli C, et al. Ethyl glucuronide hair testing: a review. Forensic Sci Int 2019;300:106-19.

32 Helander A, Péter O, Zheng Y. Monitoring of the alcohol biomarkers PEth, CDT and EtG/EtS in an outpatient treatment setting. Alcohol Alcohol 2012;47:552-7. 